\title{
Design and Development of Smart Water Purification Level Tester for Visually Impaired Person
}

\author{
Mohammad Salah Uddin ${ }^{+}$, Alinoor Hossain Olip and Md. Khabbab Hossain Tusher \\ Department of Computer Science and Engineering, East West University, Dhaka, Bangladesh.
}

\begin{abstract}
This paper presents a smart device for visually impaired persons, which helps them to determine the purification level of drinking water. Without water lives won't be exists means that water is life. The lifestyle of visually impaired person or blind people are too much challenging compared to normal people. We cannot say that, nobody can find a trouble-ness day for them. Due to their blindness they are facing huge troubles throughout their life. Smart and mobile devices are always trying to reduce their trouble-ness. In this paper we present a smart device for them for determining the purity level of drinking water based on conductivity properties. The conceptual model as well as the prototype design of the device is presented throughout this paper.
\end{abstract}

Keywords: water purification device, visually impaired, blind people, conductivity, sensors, portable device etc.

\section{Introduction}

The world is harsh for everyone but it is more difficult for persons with physical short comings. Vision Impairment is one of such a kind physical disability that many people have around the world. According to the World Health Organization (WHO), there are 253 million people living in the globe with some sort of vision impairment and around 1.1 billion people have near-vision impairment [1]. Among them 36 million are totally blind and rest of them are suffering moderate type of vision impairment [1]. Around 81 percent visually impaired people are aged 50 to above and 19 million people are suffering vision impairment whose age are less than 15 years [2]. They are facing huge amount of troubles in their daily lives, due to their blindness. They have full of emotional distress and pain [3]. Jobs sectors are not welcoming them. In Ireland only $16 \%$ of blind people are working. $41.36 \%$ and $33 \%$ blind people respectively goes for work in United Kingdom, Australia and Canada [4]. The numbers are much lower in developing countries in South Asian region. The more statistical data of visually impaired person is shown in Fig.1.

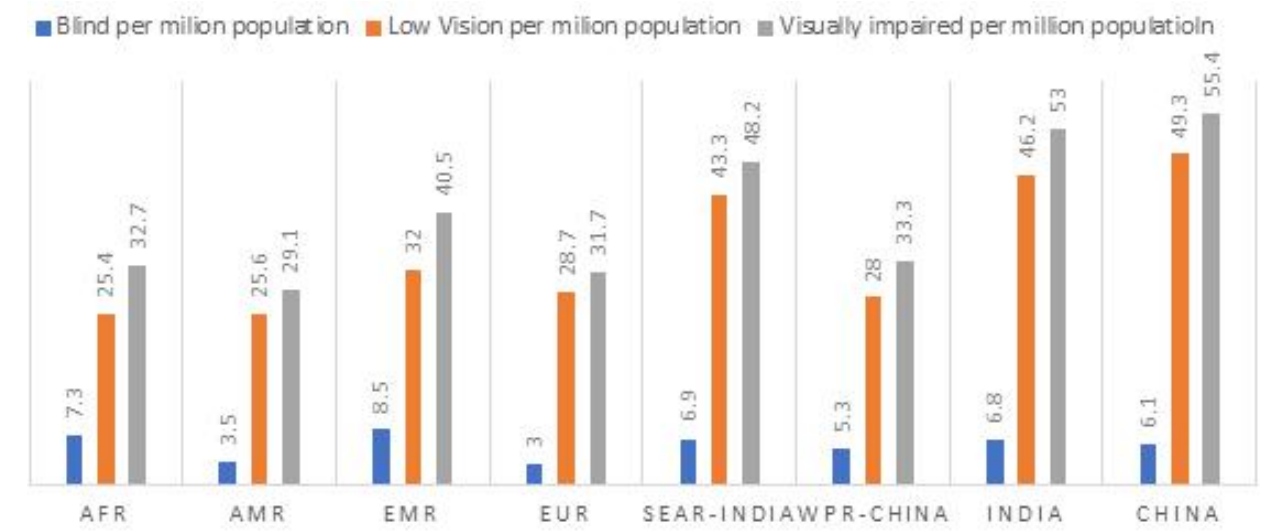

Fig. 1: Statistical data for region wise visually impaired people [2].

\footnotetext{
+ Corresponding author. Tel.: +8801818525335; Fax: Not applicable.

E-mail address: akash.bangla@gmail.com.
} 
Technology also touch their lives, makes their lives more comfortable by offering smart gadgets/tools (specially designed for them). Some of them are listed in section II. A conceptual design for measuring the purity level of drinking water is presented in this paper. The prototype implementation of the proposed system is also elaborated. Section II describe some smart devices/tools for blind people and water purification measurement device (not for blind person) are listed in section III. Section IV introduces the design and implementation phase of proposed system. The calibration and testing are also presented in this section. Conclusion is given in section $\mathrm{V}$.

\section{Existing Smart Devices for Blind People}

\subsection{VizWiz social}

VizWiz Social (in Fig.2) is an open source application (Facebook or tweeter like social apps) developed by Apple corporation with more than 5000 active users [5], specially designed for blind people. The Blind or visually impaired person can post photographs and questionnaires through audio message for requesting help (asked query) based on the posted photographs. Since, it's a social like application other users (non-blind person) help them by providing the appropriate answer through audio message. Various users (blind persons) asked for various type of questions among them 28 percent quires are related to daily foods and beverages [6].
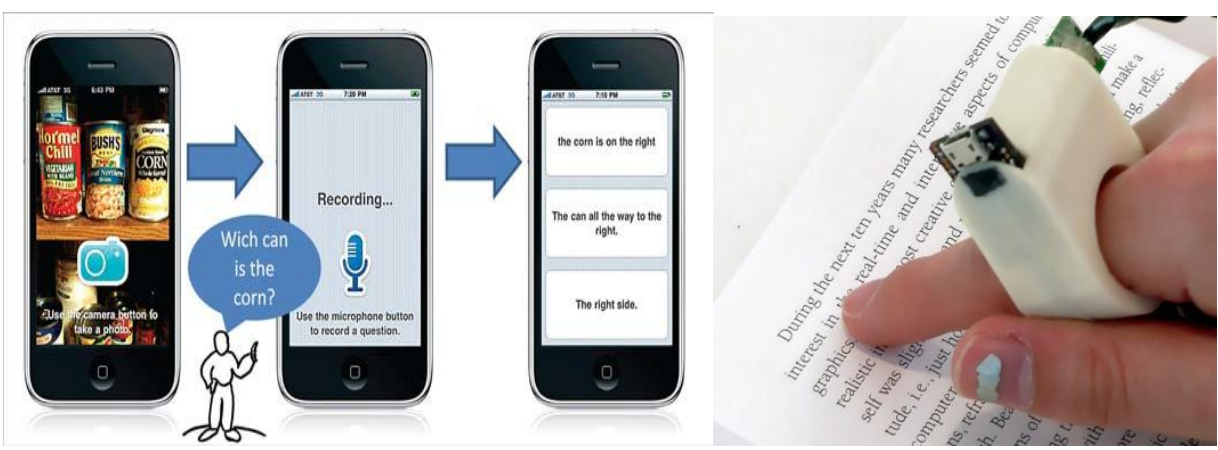

Fig. 2: VizWiz social application (left) and the finger reader device of MIT (right).

\subsection{Finger reader}

This device is in a prototype state (under research) created by the MIT Media Labs. It is a wearable device (like a finger ring, shown in Fig.2). It's a real time visual interpreting device. The device instantly read (audio output) the printed lines during the movement of the device over the printed text. The device has also a built in vibrating system which tells the users if there is any deviation off the line. The more details specifications are available on MIT's website.

\subsection{Bitlab}

BItlab is an excellent braille device made by Bitlab which is the world's first tablet for the blind people (shown in Fig.3). This device turns any texts into braille writing, which is easily recognizable by blind person. It has micro size physical bubble on screen which will automatically rise up/down depending on the contents. This device easily performs the communication with personal computer via serial port.
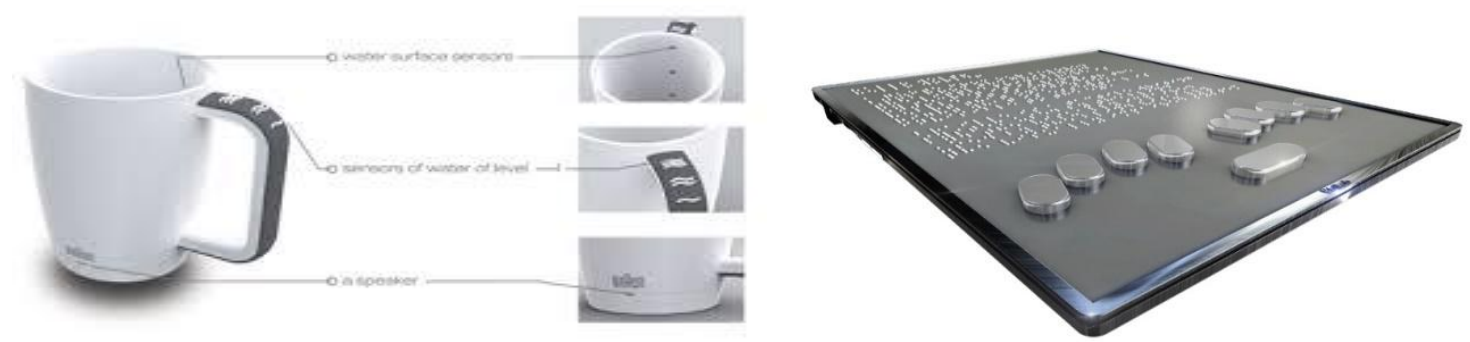

Fig. 3: The Bitlab tablet (left) and Bell mug (right).

\subsection{Bell mug}


Bell Mug (in Fig.3) is a smart mug that indicates the water level specially designed for blind persons. Blind people can set the preference level of the liquid, by using built-in indicators (only three level of indicators are available), they want to pour and when the level is achieved the mug makes an indication or beep sound.

\section{Existing Devices for Measuring Water Purification Level}

Nowadays, various devices are available for measuring the purification level of water. Almost all of them are built based on conductivity properties of water. Some of them are describe bellow:
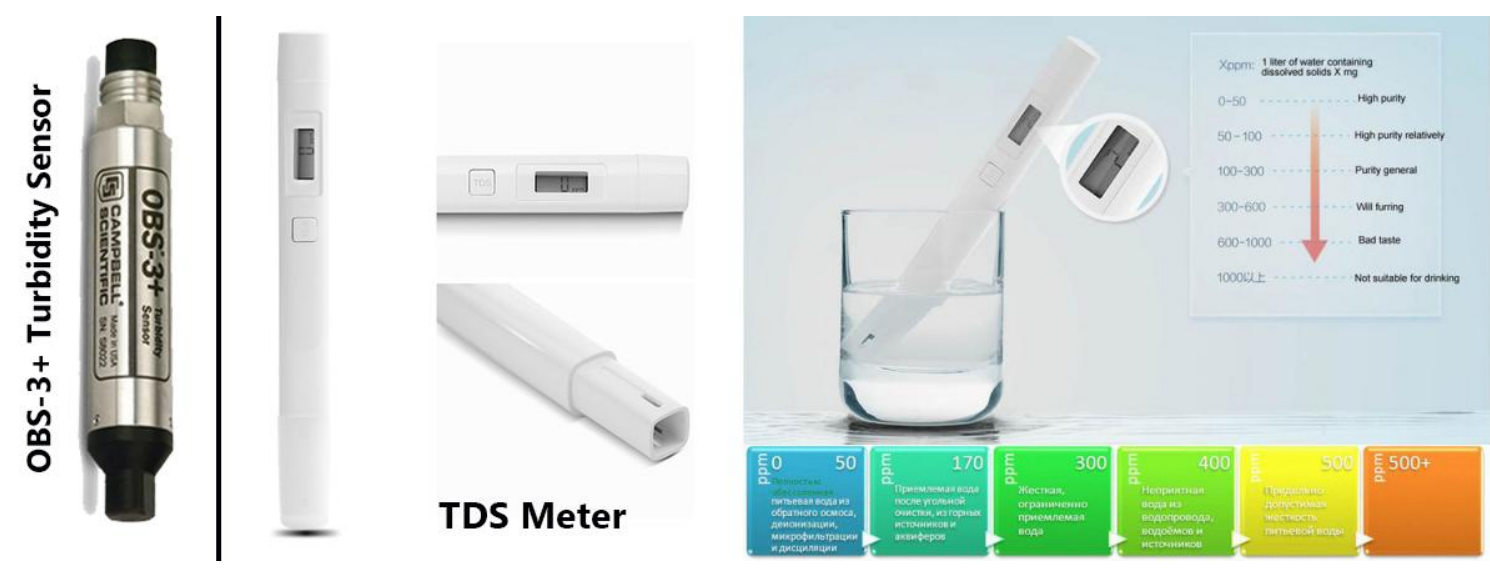

Fig. 4: The OBS-3+ Turbidity Sensor (left), TDS meter and purification level charts for TDS meter (right).

\subsection{OBS-3+ turbidity sensor}

Turbidity sensor checks the cloudiness of water (the transparent level of the water). It uses light transmittance and sees the scattering rate and then checks the turbidity (in Fig.4). Measuring turbidity level is an intelligent way for determining the water purity level.

\subsection{Xiomi TDS pen}

Anyone can able to check the water purity level by using this pen like hand-held device shown in Fig.4. It measures total dissolved solid in water through the conductivity properties of water. It gives a numerical value in ppm unit. This numerical value is then mapped with a chart for determining actual purity level. The mapping chart is also presented in Fig.4 There are several companies who sell similar types of devices.

\section{Proposed System Design and Implementation}

\subsection{Hardware and software components}

The main component of our system is designing the conductivity sensor or conductivity probe for measuring the conductivity level of water. We have chosen the pure silver wire for designing the conductivity probe. A passive element such as register $(10 \mathrm{~K})$ is connected with one wire of the probe and other terminal of the register is directly connected with ground. The connecting junction/point of register and probe is used as data point of this conductivity device. The microcontroller (in our case raspberry pi) collects data by using this point. One GPIO (General purpose input output) pins of raspberry pi is directly connected with this point for data collection. Another wire of the probe is directly connected with another GPIO's pins of raspberry pi. A simple python script is written for collecting and processing the data. The design theory of the conductivity meter is nicely elaborated in [7]. The other hardware components of our system are listed in Table -1. 

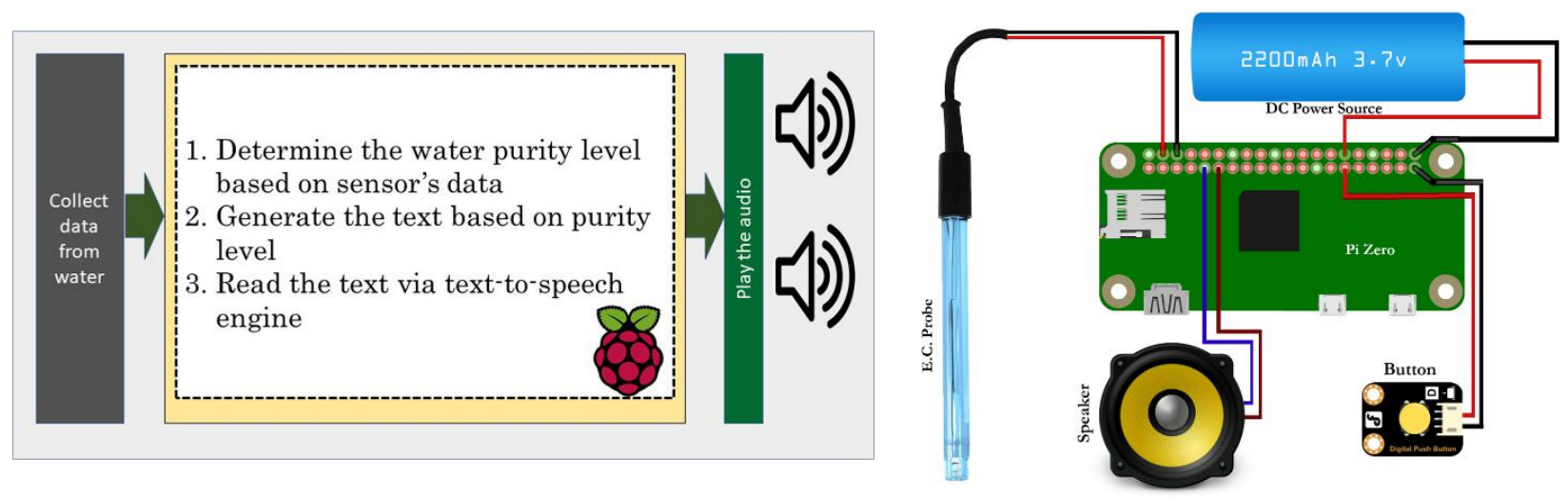

Fig. 5: The data flow diagram (left) and the circuit diagram of the system (right).

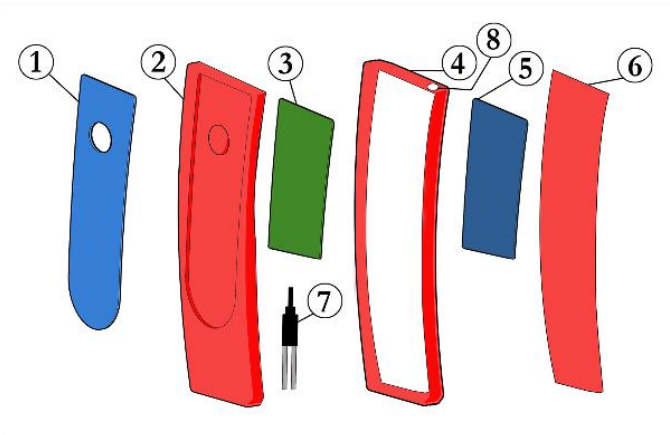

1.Front Metal cover

2. Front Housing

3. Raspberry Pi Zero

4. Housing (middle)

5. Battery

6. Back Cover

7. E.C. Probe

8. Audio out

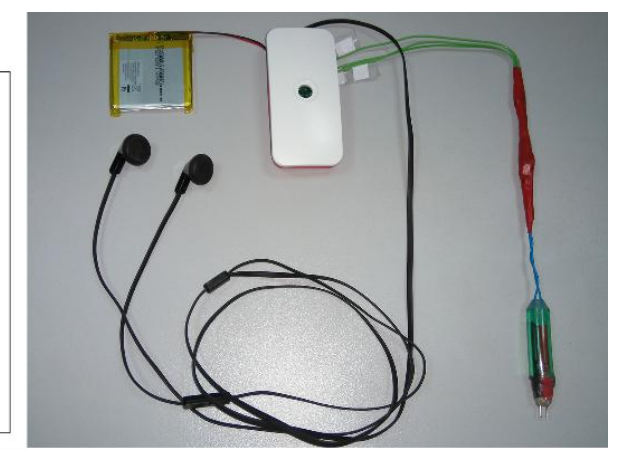

Fig. 6: The conceptual design (left) and prototype implementation of the system (right).

Table 1: The hardware components list.

\begin{tabular}{cc}
\hline Name of the components & Purpose \\
\hline Raspberry Pi Zero [8] & The main controlling board \\
\hline Speaker & For audio output \\
\hline EC. Probe & For collecting conductivity data \\
\hline Battery & Power source \\
\hline Push button & For testing/on/off \\
\hline
\end{tabular}

\subsubsection{System integration}

The whole system is integrated together with all the hardware components as shown in the circuit diagram in Fig.5. We used the open source text to speech engine for reading the text. All the source code for determining the purity level of water and interpreting the voice message is done by python script. The data flow diagram is clarified in Fig.5 and Fig.6 shows the conceptual design and prototype implementation of the proposed system.

\subsubsection{Meter calibration and testing}

The accuracy of any sensor/meter (in our case, EC probe), depends on calibration. We choose distilled water as standard pure water, since distilled water does not contain any minerals. A thermometer is needed for recording the water temperature during the calibration process. We assume that, the conductivity reading of distilled water is equal to zero. This point is chosen as zeroth point of our meter. We add some salt with water, since salted water is very conductive. The measurement value of salted water is chosen as highest 
point of the meter. Two more middle point is determined by known conductivity readings of water, which is measured by standard TDS meter.

After calibration we test our conductivity meter with different type of sample water and the result is compared with standard TDS meter's reading (available in the market). the comparison curve is shown is Fig.7.

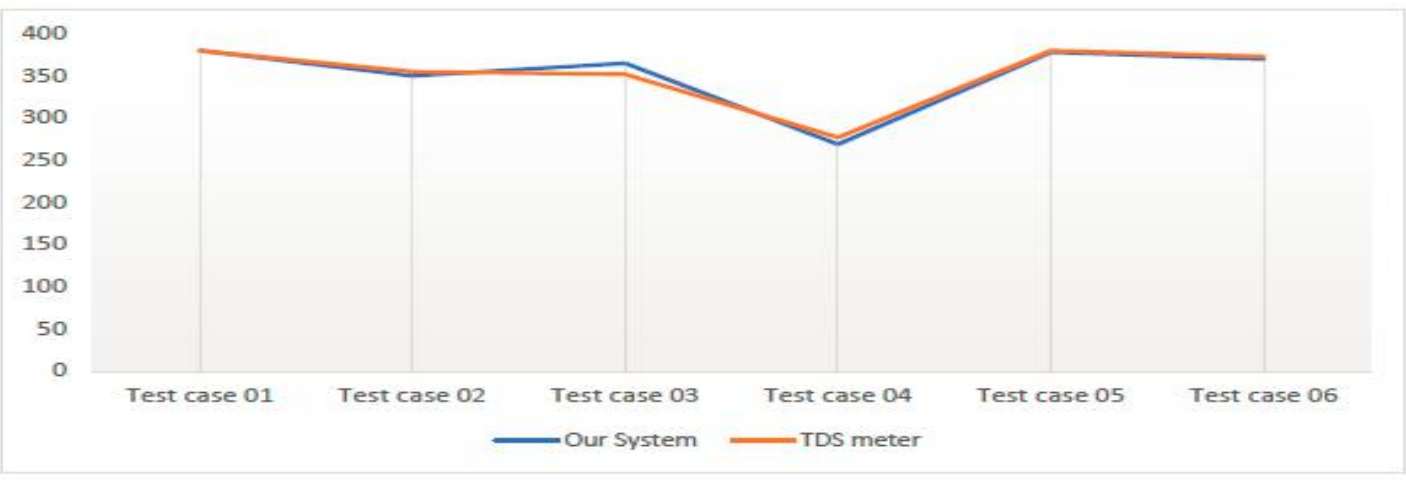

Fig. 7: The sample testing comparison between TDS meter and our system.

\section{Conclusion}

The design and implementation of water purification measurement device are presented. The conceptual device design is also included in this paper. This smart device is useful for blind people which may reduce the complexity of their lives with respect to water purification level measurement. This device is also used as a water purification tester for robotics system (service robot). We have a plan to extend this device as a liquid identification (any liquid substance) meter for blind person.

\section{References}

[1] Bourne, Rupert RA, et al. Magnitude, temporal trends, and projections of the global prevalence of blindness and distance and near vision impairment: a systematic review and meta-analysis. The Lancet Global Health 5.9 (2017): e888-e897.

[2] World Health Organization (WHO). GLOBAL DATA ON VISUAL IMPAIRMENTS 2010. World Health Organization report. 2012, WHO/NMH/PBD/12.01.

[3] Lisa Rapaport. Vision impairment linked to worse quality of life. HEALTH NEWS, www.reuters.com, September 05, 2015.

[4] Chris White, CEO, National Council for the Blind. Discrimination? 'Only $16 \%$ of people who are blind or vision impaired in Ireland are working. The journal.ie, $5^{\text {th }}$ June 2017.

[5] Brady, Erin L., et al. Investigating the appropriateness of social network question asking as a resource for blind users. Proceedings of the 2013 conference on Computer supported cooperative work. ACM, 2013.

[6] Brady, Erin, et al. Visual challenges in the everyday lives of blind people. Proceedings of the SIGCHI Conference on Human Factors in Computing Systems. ACM, 2013.

[7] Emily Gertz, P Patrick Di Justo. Environmental Monitoring with Arduino. O’Reilly Media, Inc.2012.

[8] RASPBERRY PI FOUNDATION UK. Raspberry Pi Zero. 2015 